\title{
Determination of Volatile Components of Helichrysum arenarium subsp. aucheri Naturally Distributed in Two Different Regions
}

\author{
Ebru Hatice Tığlı Kaytanlıŏglu ${ }^{*}$, Sevgin Özderin ${ }^{2}$, Hüseyin Fakir ${ }^{3}$, Emre Gümüşay ${ }^{4}$

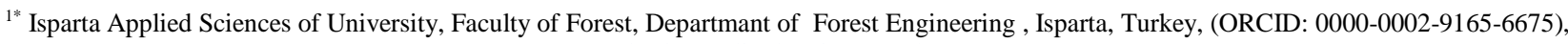 \\ ebrukaytanlioglu@isparta.edu.tr \\ ${ }^{2}$ Muğla Sitkı Koçman University, Köyceğiz Vocational High School, Departmant of Forestry, Muğla, Turkey, (ORCID: 0000-0002-4511-5229), \\ sevginozderin@mu.edu.tr \\ ${ }^{3}$ Isparta Applied Sciences of University, Faculty of Forest, Departmant of Forest Engineering , Isparta, Turkey, (ORCID: 0000-0002-6606-8011), \\ huseyinfakir@isparta.edu.tr \\ ${ }^{4}$ Isparta Applied Sciences of University, Faculty of Forest, Departmant of Forest Engineering, Isparta, Turkey, (ORCID: 0000-0002-6108-5373), \\ gumus_emre01@hotmail.com
}

(First received 27 February 2021 and in final form 27 June 2021)

(DOI: $10.31590 /$ ejosat.887605)

ATIF/REFERENCE: Tığlı Kaytanlığlu, E.H., Özderin, S., Fakir, H. \& Gümüşay, E. (2021). Determination of Volatile Components of Helichrysum arenarium subsp. aucheri Naturally Distributed in Two Different Regions. European Journal of Science and Technology, (25), 152-158.

\begin{abstract}
The aim of this study was to determine the volatile components of Helichrysum arenarium (L.) Moench subsp. aucheri that belongs to the Helichrysum genus, belonging to the Asteraceae family, one of the important families for Turkey and generally known as 'ölmez çiçek, altın otu or mantuvar' in Turkey. To determine volatile components, leaves and flowers of Helichrysum arenarium subsp. aucheri specimens were collected from Isparta Aksu and Adana Feke villages in Turkey, and dried at room temperature. Then, volatile components were determined with HS-SPME/GC-MS analysis. As a result of the findings obtained, a total of 86 volatile components were found for Helichrysum species. A total of 64 volatile components were determined for Helichrysum arenarium subsp. aucheri specimens collected from Isparta Aksu region, whereas the main components were found as trans-Caryophyllene

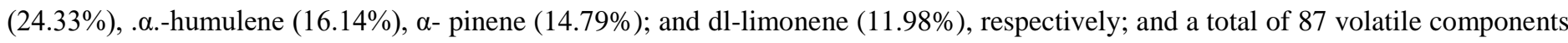
for specimens collected from Adana Feke region, whereas the main components were found as trans-caryophyllene (24.57\%), $\alpha$ pinene (22.5\%), $\beta$-pinene (8.98\%), limonene (8.21\%), respectively.
\end{abstract}

Keywords: Helichrysum arenarium subsp. aucheri, volatile components, HS-SPME/GC-MS, $\alpha$ - pinene, trans-caryophyllene, Türkiye

\section{İki Farklı Yörede Doğal Yayılış Gösteren Yayla Çiçeği (Helichrysum arenarium subsp. aucheri)'nin Uçucu Bileșenlerinin Belirlenmesi}

$\ddot{\text { Öz }}$

Bu çalı̧̧mada ülkemiz için önemli familyalarından olan Asteraceae familyasına ait Türkiye'de yaygın olarak 'ölmez çiçek, altın otu veya mantuvar'olarak bilinen Helichrysum cinsine ait Helichrysum arenarium (L.) Moench subsp. aucheri'nin uçucu bileşenlerinin belirlenmesi amaçlanmıştır. Isparta Aksu ve Adana Feke Köyü mevkiilerinden doğal olarak yayılış gösteren Helichrysum arenarium subsp. aucheri örnekleri uçucu bileşenleri belirlemek amacıyla yaprak ve çiçekleri toplanmış ve oda sıcaklı̆ğında kurutulmuştur. Daha sonra HS-SPME/GC-MS analizi ile uçucu bileşenleri belirlenmiştir. Elde edilen bulgular sonucunda altın otu türüne ait toplam 86 adet uçucu bileşen belirlenmiştir. Isparta Aksu mevkiinden toplanan Helichrysum arenarium subsp. aucheri 'da 64 uçucu bileşen, bu bileşenler içerisinde temel bileşenler; trans-caryophyllene $\% 24.33, \alpha$-humulene $\% 16.14, \alpha$-pinene $\% 14.79$; dl-limonene (\% 11.98); Adana Feke Köyü mevkii örneklerinde 87 uçucu bileşen, bu bileşenler içerisinde temel bileşenler; trans-caryophyllene (\%24.57), $\alpha$ pinen(22.5), $\beta$-pinen (\% 8.98), limonene (\%8.21) bileşenleri tespit edilmiştir.

Anahtar Kelimeler: Helichrysum arenarium subsp. aucheri, uçucu bileşen, HS-SPME/GC-MS, $\alpha$-Pinene, trans-Caryophyllene, Türkiye. 


\section{Introduction}

Thanks to its geographical location, climate and plant diversity, agricultural potential, and wide area, Turkey is one of the leading countries in the trade of medicinal and aromatic plants, because of its geographical location. This importance of Turkey stems from the fact that plants that produce many herbal products constituting the input of herbal medicine, plant chemicals, food and additive agents, and cosmetics and perfume industries present in the natural flora of Turkey. Therefore, these plants are being marketed with the collection from the wild (Bayram et al., 2010). Medicinal and aromatic plants have been used in health promotion and disease prevention. By observing the protection methods developed by the defense mechanisms of plants by secreting active substances such as various enzymes, essential fatty acids, and phenolic substances, it was identified with the trial-and-error method that these have been good for the treatment of a number of ailments in humans (Kayaalp, 2001). Growing production and marketing of natural health-promotion and personal care products has also created an increasing industrial demand for medicinal and aromatic plants as well (Igwillo, 2019). Medicinal and aromatic plants contain bioactive secondary metabolites like steroids, flavonoids, saponins, alkaloids, terpenes, and phenolic compounds. These secondary metabolites possess antimicrobial, antifungal, antiallergic, antidiabetic, cardioprotective, antioxidant, anticancer, antithyroid, antihistaminic, antimalarial, anthelminthic, antiinflammatory, antihypertensive, antispasmodic, and analgesic properties (Aftab, 2019). Medicinal and aromatic plants can be found in fresh, frozen, or dry forms. These plants are preferred in the pharmaceutical industry because of their therapeutic effects. Fixed oils (or fats, fatty acids that plants have (might be added) and essential oils extracted from plants are used in soft drinks and candies within food industry and in perfumes, skin care and hair care products and aromatherapy within cosmetic industry (Van Vuuren et al., 2010, Christaki, 2012). Since they are complex mixtures, effectiveness of essential oils varies depending on the amount and type of substances they contain (Bayaz, 2014).

The fact that almost 5.000 of the 7.000 chemical compounds, of which have been isolated and identified from plants thus far, were extracted from the members of this family is an indicator of how rich they are in chemical terms (Zeybek and Zeybek, 2002). The genus Helichrysum consists of an estimated 600 species all around the world, and is widely distributed in South Africa (250 species), Southern Europe, Southern-Western Asia, Southern India, Sri Lanka, Australia, and the Mediterranean basin (Anderberg, 1991). This genus is represented by 27 taxa, 15 of which are endemic in Turkish flora (Davis, et al., 1988; Güner et al., 2000; Sümbül, et al., 2003). Helichrysum species are grown in every region of Turkey and is supplied fresh in every season as it is not affected by the climate conditions. Hel species have been used as Daily herbal tea in Turkey, since they have bile and diuretic effects. (Şen and Kalayc1, 2016).

Heli species, growing in Corsica, France, is one of the most preferred species in perfumery and cosmetics industry around the World. (Bianchini et al., 2001). The economically used part of the Helichrysum species is its flowers. The main active substance of yellow flowers of Helichrysum species is the yellowish-colored essential oils in glandular hairs (Licina and
Kralj, 2016). Despite its floral scent, it can be used as a base note in perfume blends thanks to its strength and color (Lawless, 2002). Helichrysum species are reported to have a high degree of polymorphism (Peyron and Roubaud, 1971). Previous studies have reported that some of them show significant pharmacological properties and find wide use in perfumery (Bianchini et al., 2001). It was identified that Helichrysum volatile components show anti-inflammatory (Sala et al., 2002; Appendino et al., 2007), antimicrobial (Roussis et al., 1998; Nostro et al., 2001; Angioni et al., 2003), and antioxidant (Sala et al., 2002) activities. Helichrysum volatile components reportedly have anti-inflammatory, analgesic, cell regenerative, pain reducing, and sedative effects and it relieves stress. By reason of the fact that "anti-aging skincare" comes to mind when it comes to cell regeneration recently; Helichrysum volatile components are dermatologically applied against cracked skins, hemorrhoids, acne scars, surgical scars, and wounds, and can be in-depth effective even with a small content of dilutions (Harris and Harris, 2002; Haas, 2004; Schnaubelt, 2011). Helichrysum volatile components have been reported to be of great value as a cell regenerative and decongestant thanks to the fact that neryl acetate, one of the components in essential oil, has a very high antiradical activity and increases collagen type I production about 6 times (Millou et al., 2010). As they can aid the renewal of skin cells, they are used in skincare products and called "liquid stitches" (Price and Price, 2012). Additionally, studies suggest that Helichrysum volatile components prevent blood clot formation and accumulation (Marković, 2005; Battaglia, 2003; Gattefossé, 1993) and have mucolytic, anti-spasmodic, and expectorant effects, which are helpful for coughs, bronchitis, and sinusitis (Battaglia, 2003; Lawless, 2002; Poštić, 2013). Besides, it is reported that Helichrysum volatile components, together with chamomile (Chamaemelum nobile) and yarrow (Achillea millefolium), are used to reduce fever and has an antiinflammatory effect (Mojay, 1996). It is reported that Helichrysum hydrosol, which is obtained by steam distillation, can help to relieve painful menstrual cramps, cleanse the liver from toxins, and combat gingivitis, as a mouthwash (Catty, 2001).

SPME (solid-based micro-extraction method) saves processing time and costs as it is a method that combines sample preparation, extraction and concentration stages in a single solvent-free stage. However, positive developments were observed in the sample preparation phase and results. The type and thickness of the material covering the fiber part in the syringe affects the effectiveness of the SPME method. The fact that SPME method can be performed in a short time such as 130 minutes reveals its advantage over other methods (Vas \& Vekey, 2004; Araujo et al., 2007; Dönmez \& Salman, 2017).

The aim of this study was to determine volatile components that were extracted from Helichrysum arenarium (L.) Moench subsp. aucheri (Boiss.) P.H. Davis \& Kupicha, using solid-phase microextraction (SPME, Supelco, Germany) procedure.

\section{Material and Method}

\subsection{Material}

Helichrysum arenarium subsp. aucheri specimens, the research material, were collected from the research area in Aksu

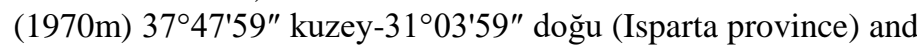

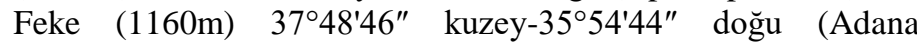
province) counties in Turkey within the vegetation period. 
Helichrysum arenarium subsp. aucheri species constitute samples of leaves and flowers. The plant was diagnosed by us using the discrimination key in "Flora of Turkey" (Davis, 1982).

The voucher specimens were placed in sample bags and the bags were labeled after coding, and collection data (collection time, place, and elevation) were marked on the label. The plant samples were kept at room temperature in a semi-dark and airy place, to use in the analysis of volatile components. After the specimens were dried, they were brought to the Laboratory of the Department of Forest Botany, Faculty of Forestry, Isparta University of Applied Sciences. The plant samples were identified in the Herbarium at S. Demirel University, Faculty of Arts and Science, Department of Biology. The identified voucher specimens were deposited in the Herbarium at Isparta University of Applied Sciences, Faculty of Forestry.

\subsection{Determination of leaf and flower volatile components with HS-SPME/GC-MS analysis}

In this study, leaf and flower samples were collected from the area where Helichrysum species grow, within the vegetation period. The collected leaf and flower samples were placed in bags and brought to the Laboratory of the Department of Forest Botany, Faculty of Forestry, Isparta University of Applied

\section{Results}

In this study, volatile components of Helichrysum arenarium subsp. aucheri specimens were determined with SPME (Solid-Phase Microextraction) analysis. The volatile components determined were given in Table 1.

As a result of SPME analyses, a total of 64 volatile components were determined for Helichrysum arenarium subsp.
Sciences within the same day, without any delay and exposure to sunlight. The voucher specimens were dried at room temperature $\left(25^{\circ} \mathrm{C}\right)$ to constant weight.

Floral scent components of flowers and leaves were combined with Gas Chromatography/ Mass Spectrometric (GCMS) and determined with Headspace Solid-Phase Microextraction (HS-SPME) procedure. Based on solid-phase microextraction (SPME, Supelco, Germany) procedure, $2 \mathrm{~g}$ of flower and leaf specimens were placed into a $10 \mathrm{~mL}$ vial and heated to $60^{\circ} \mathrm{C}$ for 30 minutes. After that, volatile components were absorbed from headspace using a $75 \mu \mathrm{m}$-thick Carboxen/Polydimethylsiloxane (CAR/PDMS) coated fused silica fiber and injected into capillary column (Restek Rx-5 Sil MS $30 \mathrm{~m} \times 0.25 \mathrm{~mm}, 0.25 \mu \mathrm{m}$ ) of HS-SPME-compatible GCMS (Shimadzu 2010 PLUS) instrument. The oven temperature was set to keep at $40^{\circ} \mathrm{C}$ for 2 minutes and to reach $250^{\circ} \mathrm{C}$ with a $4^{\circ} \mathrm{C}$ increase per minute. Injection and detection temperatures were set at $250^{\circ} \mathrm{C}$. EI $(70 \mathrm{eV})$ was used as an ionization mode and Helium (1.61 mL per minute) was used as carrier gas. Wiley, NIST Tutor, and FFNSC libraries were used to identify volatile components. LRI (Linear Retention Indices) values were calculated by using a series of the standards of C7-C30 saturated $n$-alkanes (Sigma-Aldrich Chemical Co., USA).

aucheri specimens collected from Isparta Aksu area, whereas the main components were found as trans-Caryophyllene $(24.33 \%)$,

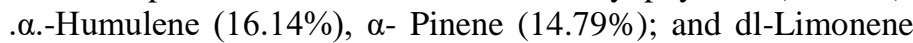
$(11.98 \%)$, respectively. Similarly, a total of 86 volatile components were determined for Helichrysum arenarium subsp. aucheri specimens collected from Adana Feke village, whereas the main components were found as trans-Caryophyllene (24.57\%), $\alpha$-Pinene (22.5\%), $\beta$-Pinene $(8.98 \%)$, Limonene $(8.18 \%)$, respectively.

Table 1. Volatile components of Helichrysum arenarium subsp. aucheri

\begin{tabular}{|c|c|c|c|c|c|}
\hline R.T. & Components & $\begin{array}{c}\text { Flowering } \\
\text { (Adana) }\end{array}$ & $\begin{array}{c}\text { Flowering } \\
\text { (Isparta) }\end{array}$ & Formula & Category \\
\hline 4.656 & Hexanal & - & 1.62 & $\mathrm{C}_{6} \mathrm{H}_{12} \mathrm{O}$ & AA \\
\hline 6.144 & 2-Hexenal & - & 1.23 & $\mathrm{C}_{6} \mathrm{H}_{10} \mathrm{O}$ & AA \\
\hline 6.228 & 7-Methyl-1-Octene & - & 0.98 & $\mathrm{C}_{9} \mathrm{H}_{18}$ & $\mathrm{OC}$ \\
\hline 6.341 & Ethyl-Benzene & - & 0.12 & $\mathrm{C}_{8} \mathrm{H}_{10}$ & $\mathrm{AH}$ \\
\hline 6.630 & 1,2-Dimethyl-Benzene & - & 0.16 & $\mathrm{C}_{8} \mathrm{H}_{10} \mathrm{O}_{2}$ & $\mathrm{AH}$ \\
\hline 6.671 & 1,4-Dimethyl-Benzene & - & 0.08 & $\mathrm{C}_{8} \mathrm{H}_{10} \mathrm{O}_{2}$ & $\mathrm{AH}$ \\
\hline 7.332 & Styrene & - & 0.37 & $\mathrm{C}_{8} \mathrm{H}_{8}$ & $\mathrm{OC}$ \\
\hline 7.400 & 1-Nonanol & - & 0.38 & $\mathrm{C}_{9} \mathrm{H}_{20} \mathrm{O}$ & $\mathrm{OC}$ \\
\hline 7.754 & Heptanal & - & 0.09 & $\mathrm{C}_{7} \mathrm{H}_{14} \mathrm{O}$ & AA \\
\hline 7.804 & Bornylene & - & 0.09 & $\mathrm{C}_{10} \mathrm{H}_{16}$ & $\mathrm{MH}$ \\
\hline 8.410 & Tricyclo heptane & - & 0.02 & $\mathrm{C} 7 \mathrm{H} 10$ & $\mathrm{AH}$ \\
\hline 8.426 & Tricyclene & 0.05 & - & $\mathrm{C}_{10} \mathrm{H}_{16}$ & MH \\
\hline 8.755 & 3-Heptanone & - & 0.03 & $\mathrm{C}_{7} \mathrm{H}_{14} \mathrm{O}$ & MH \\
\hline 8.911 & $\alpha$-Pinene & 22.15 & 14.79 & $\mathrm{C}_{10} \mathrm{H}_{16}$ & MH \\
\hline 9.361 & a.-Fenchene & - & 0.54 & $\mathrm{C}_{10} \mathrm{H}_{16}$ & MH \\
\hline 9.369 & $\begin{array}{l}\text { 2,2-Dimethyl-3- } \\
\text { Methylene }\end{array}$ & 0.23 & - & $\mathrm{C}_{11} \mathrm{H}_{22}$ & $\mathrm{MH}$ \\
\hline 9.421 & Camphene & 0.95 & 0.75 & $\mathrm{C}_{11} \mathrm{H}_{22}$ & MH \\
\hline 9.739 & 4-Undecene & 0,25 & 0.34 & $\mathrm{C}_{11} \mathrm{H}_{22}$ & $\mathrm{AH}$ \\
\hline 9.859 & Benzaldehyde & 0.21 & 0.29 & $\mathrm{C}_{7} \mathrm{H}_{6} \mathrm{O}$ & AAI \\
\hline 9.950 & Cyclopropane & 0.22 & 0.16 & $\mathrm{C}_{3} \mathrm{H}_{6}$ & $\mathrm{SH}$ \\
\hline 10.094 & 2-Hexenoic Acid & 0.04 & - & $\mathrm{C}_{7} \mathrm{H}_{12} \mathrm{O}_{2}$ & FA \\
\hline 10.235 & Cymene & 0.03 & - & $\mathrm{C}_{10} \mathrm{H}_{14}$ & $\mathrm{MH}$ \\
\hline 10.502 & 2-. $\beta$-Pinene & 8.98 & 6.38 & $\mathrm{C}_{10} \mathrm{H}_{16}$ & MH \\
\hline 10.680 & 1-Octen-3-Ol & 0.08 & 0.04 & $\mathrm{C}_{8} \mathrm{H}_{16} \mathrm{O}$ & AAI \\
\hline 10.839 & 6-Methyl-5-Hepten-2-One & 0.07 & 0.40 & $\mathrm{C}_{8} \mathrm{H}_{14} \mathrm{O}$ & AAI \\
\hline 11.004 & $\beta$-Myrcene & 0.87 & 4.83 & $\mathrm{C}_{10} \mathrm{H}_{16}$ & SH \\
\hline
\end{tabular}


European Journal of Science and Technology

\begin{tabular}{|c|c|c|c|c|c|}
\hline 11.080 & $\begin{array}{l}\text { Benzoic Acid 1-Methyl-Heptyl } \\
\text { Ester }\end{array}$ & - & 0.10 & $\mathrm{C}_{15} \mathrm{H}_{20} \mathrm{O}_{2}$ & AAI \\
\hline 11.090 & 1-Decene & 0.13 & - & $\mathrm{C}_{10} \mathrm{H}_{20}$ & MH \\
\hline 11.288 & $\begin{array}{l}\text { Methyl 12- } \\
\text { Methyltetradecanoate }\end{array}$ & 0.13 & - & $\mathrm{C}_{16} \mathrm{H}_{32} \mathrm{O}_{2}$ & $\mathrm{OC}$ \\
\hline 11.289 & Methyl Isohexanoate & - & 0.15 & $\mathrm{C}_{7} \mathrm{H}_{14} \mathrm{O}_{2}$ & AAI \\
\hline 11.350 & Trans-2-Furan & 0.02 & 0.05 & $\mathrm{C}_{9} \mathrm{H}_{12} \mathrm{O}$ & $\mathrm{OC}$ \\
\hline 11.530 & Octanal & 0.02 & 0.13 & $\mathrm{C}_{8} \mathrm{H}_{16} \mathrm{O}$ & $\mathrm{AA}$ \\
\hline 11.588 & L-Phellandrene & 0.23 & 0.21 & $\mathrm{C}_{10} \mathrm{H}_{16}$ & $\mathrm{MH}$ \\
\hline 11.678 & Linalyl Acetate & - & 0.14 & $\mathrm{C}_{12} \mathrm{H}_{20} \mathrm{O}_{2}$ & $\mathrm{OM}$ \\
\hline 11.685 & 4.-3-Carene & 0.17 & - & $\mathrm{C}_{10} \mathrm{H}_{16}$ & MH \\
\hline 11.851 & 1,4-Dichlorobenzene & 0.23 & 0.18 & $\mathrm{C}_{10} \mathrm{H}_{12}$ & $\mathrm{AH}$ \\
\hline 12.011 & a.-Terpınene & 0.62 & 0.70 & $\mathrm{C}_{10} \mathrm{H}_{16}$ & $\mathrm{MH}$ \\
\hline 12.297 & 1-Methyl-4 benzene & - & 1.79 & $\mathrm{C}_{10} \mathrm{H}_{12}$ & MH \\
\hline 12.302 & Methyl -benzene & 0.60 & - & $\mathrm{C}_{10} \mathrm{H}_{14}$ & $\mathrm{AH}$ \\
\hline 12.503 & dl-Limonene & - & 11.98 & $\mathrm{C}_{10} \mathrm{H}_{16}$ & $\mathrm{MH}$ \\
\hline 12.523 & Limonene & 8.21 & - & $\mathrm{C}_{10} \mathrm{H}_{16}$ & MH \\
\hline 12.608 & 1,8-Cineole & 0.18 & 1.27 & $\mathrm{C}_{10} \mathrm{H}_{18} \mathrm{O}$ & $\mathrm{MH}$ \\
\hline 12.803 & Cis-Ocimene & 0.79 & 0.04 & $\mathrm{C}_{10} \mathrm{H}_{16}$ & MH \\
\hline 12.895 & Oct-3(E)-En-2-One & 0.01 & - & $\mathrm{C}_{8} \mathrm{H}_{14} \mathrm{O}$ & AAI \\
\hline 13.008 & Benzene acetaldehyde & 0.06 & 0.08 & $\mathrm{C}_{8} \mathrm{H}_{8} \mathrm{O}$ & AAI \\
\hline 13.206 & 1,3,6-Octatriene & 1.14 & 0.10 & $\mathrm{C}_{8} \mathrm{H}_{12}$ & $\mathrm{AH}$ \\
\hline 13.381 & $\begin{array}{l}\text { 2-Isopropenyl-5-Methylhex-4- } \\
\text { Enal }\end{array}$ & 0.01 & - & $\mathrm{C}_{10} \mathrm{H}_{18} \mathrm{O}$ & AAI \\
\hline 13.626 & 1,4-Cyclohexadiene & 0.97 & 0.87 & $\mathrm{C}_{10} \mathrm{H}_{16}$ & MH \\
\hline 14.087 & 3,5-Octadien-2-One & 0.06 & 0.05 & $\mathrm{C}_{8} \mathrm{H}_{12} \mathrm{O}$ & AAI \\
\hline 14.490 & Ethenyl Benzene & 0.02 & - & $\mathrm{C}_{8} \mathrm{H}_{10}$ & $\mathrm{AH}$ \\
\hline 14.684 & $\alpha$-Terpinolene & 1.06 & 0.60 & $\mathrm{C}_{10} \mathrm{H}_{16}$ & MH \\
\hline 14.844 & $\begin{array}{l}\text { 1-Methyl-4- } \\
\text { İsopropenylbenzene }\end{array}$ & - & 0.25 & $\mathrm{C}_{10} \mathrm{H}_{12}$ & MH \\
\hline 14.846 & 1-Isopropenylbenzene & 0.16 & - & $\mathrm{C}_{12} \mathrm{H}_{16}$ & $\mathrm{AH}$ \\
\hline 14.981 & Cyclopropane & 0.13 & - & $\mathrm{C} 3 \mathrm{H} 6$ & $\mathrm{AH}$ \\
\hline 15.285 & Linalool & 0.04 & - & $\mathrm{C}_{10} \mathrm{H}_{18} \mathrm{O}$ & $\mathrm{OM}$ \\
\hline 15.461 & Nonanal & 0.08 & 0.45 & $\mathrm{C}_{9} \mathrm{H}_{18} \mathrm{O}$ & AAI \\
\hline 15.807 & Neryl Nitrile & - & 0.05 & $\mathrm{C}_{10} \mathrm{H}_{15} \mathrm{~N}$ & $\mathrm{OC}$ \\
\hline 15.809 & Cyclohexane & 0.04 & - & $\mathrm{C}_{6} \mathrm{H}_{12}$ & $\mathrm{AH}$ \\
\hline 16.216 & Octanoic Acid & 0.08 & - & $\mathrm{C}_{8} \mathrm{H}_{16} \mathrm{O}_{2}$ & AAI \\
\hline 16.824 & 2,4,6-Octatriene & 0.07 & - & $\mathrm{C}_{10} \mathrm{H}_{16}$ & $\mathrm{AH}$ \\
\hline 17.026 & Heptan-2-One & - & 0.11 & $\mathrm{C}_{7} \mathrm{H}_{14} \mathrm{O}$ & $\mathrm{OC}$ \\
\hline 17.300 & 2,6-Nonadienal & 0.02 & - & $\mathrm{C}_{9} \mathrm{H}_{14} \mathrm{O}$ & AAI \\
\hline 17.469 & 1-Undecene & 0.06 & - & $\mathrm{C}_{11} \mathrm{H}_{22}$ & $\mathrm{AH}$ \\
\hline 17.581 & 2-Nonenal & 0.02 & 0.14 & $\mathrm{C}_{9} \mathrm{H}_{14} \mathrm{O}$ & AAI \\
\hline 18.352 & 3-Cyclohexen-1-Ol & 0.03 & - & $\mathrm{C}_{9} \mathrm{H}_{14} \mathrm{O}$ & $\mathrm{AH}$ \\
\hline 18.356 & Trans-Sabinene Hydrate & - & 0.09 & $\mathrm{C}_{10} \mathrm{H}_{18} \mathrm{O}$ & $\mathrm{OM}$ \\
\hline 18.766 & Benzoic Acid & 0.04 & - & $\mathrm{C}_{7} \mathrm{H}_{6} \mathrm{O}_{2}$ & AAI \\
\hline 18.921 & $\beta .-F e n c h y l$ Alcohol & 0.09 & 0.13 & $\mathrm{C}_{10} \mathrm{H}_{18} \mathrm{O}$ & $\mathrm{OM}$ \\
\hline 19.010 & 1-methoxy-4 benzene & 0.12 & 0.06 & $\mathrm{C}_{6} \mathrm{H}_{6}$ & $\mathrm{AH}$ \\
\hline 19.350 & Decanal & - & 0.08 & $\mathrm{C}_{10} \mathrm{H}_{20} \mathrm{O}$ & $\mathrm{OM}$ \\
\hline 20.010 & Nonanoic Acid & 0.05 & - & $\mathrm{C}_{9} \mathrm{H}_{18} \mathrm{O}_{2}$ & AAI \\
\hline 20.263 & $\begin{array}{l}\text { Z-3-Hexenyl 2- } \\
\text { Methylbutanoate }\end{array}$ & 0.07 & - & $\mathrm{C}_{11} \mathrm{H}_{20} \mathrm{O}_{2}$ & AAI \\
\hline 20.469 & Butanoic Acid & 0.15 & - & $\mathrm{C}_{4} \mathrm{H}_{8} \mathrm{O}_{2}$ & MH \\
\hline 20.550 & $\begin{array}{l}\text { 2-Isopropyl-1-Methoxy-4- } \\
\text { Methylbenzene }\end{array}$ & 0.05 & - & $\mathrm{C}_{11} \mathrm{H}_{16} \mathrm{O}$ & $\mathrm{AH}$ \\
\hline 20.693 & Butanoate & 0.02 & - & $\mathrm{C}_{8} \mathrm{H}_{16} \mathrm{O}$ & AAI \\
\hline 20.981 & Linalyl Acetate & 0.06 & - & $\mathrm{C}_{12} \mathrm{H}_{20} \mathrm{O}_{2}$ & $\mathrm{OM}$ \\
\hline 22.214 & Exobornyl Acetate & 0.07 & - & $\mathrm{C}_{12} \mathrm{H}_{20} \mathrm{O}$ & $\mathrm{OM}$ \\
\hline 22.769 & Carvacrol & 0.04 & - & $\mathrm{C}_{10} \mathrm{H}_{14} \mathrm{O}$ & $\mathrm{OM}$ \\
\hline 23.589 & 3,7-Cycloundecadien-1-Ol & 0.01 & - & $\mathrm{C}_{15} \mathrm{H}_{26} \mathrm{O}$ & $\mathrm{OC}$ \\
\hline 25.204 & Cyclosativene & - & 0.31 & $\mathrm{C}_{15} \mathrm{H}_{24}$ & $\mathrm{SH}$ \\
\hline 25.231 & Ylangene & 3.22 & - & $\mathrm{C}_{15} \mathrm{H}_{24}$ & $\mathrm{SH}$ \\
\hline 25.457 & a.-Copaene & 3.61 & 2.86 & $\mathrm{C}_{15} \mathrm{H}_{24}$ & $\mathrm{SH}$ \\
\hline 25.576 & Satıven & 0.20 & - & - & $\mathrm{OC}$ \\
\hline 26.345 & Tetradecane & 0.03 & 0.21 & $\mathrm{C}_{16} \mathrm{H}_{34}$ & $\mathrm{AH}$ \\
\hline 26.426 & Undec-4-Ene & 0.12 & - & $\mathrm{C}_{15} \mathrm{H}_{24}$ & $\mathrm{SH}$ \\
\hline 26.430 & Caryophyllene & - & 0.09 & $\mathrm{C}_{15} \mathrm{H}_{24}$ & $\mathrm{SH}$ \\
\hline 26.527 & a.-Gurjunene & 0.26 & 0.04 & $\mathrm{C}_{15} \mathrm{H}_{24}$ & $\mathrm{SH}$ \\
\hline 26.650 & Nonane & 0.03 & - & $\mathrm{C}_{9} \mathrm{H}_{20}$ & $\mathrm{AH}$ \\
\hline 26.788 & Dehydro aromadendrane & 0.04 & - & $\mathrm{C}_{15} \mathrm{H}_{24}$ & $\mathrm{SH}$ \\
\hline 27.034 & Trans-Caryophyllene & 24.57 & 24.33 & $\mathrm{C}_{15} \mathrm{H}_{24}$ & $\mathrm{SH}$ \\
\hline 27.265 & Ep1-Bicyclosesquiphellandrene & 0.03 & - & $\mathrm{C}_{10} \mathrm{H}_{16}$ & $\mathrm{OC}$ \\
\hline 27.456 & a.-Guaiene & 0.06 & - & $\mathrm{C}_{15} \mathrm{H}_{24}$ & $\mathrm{SH}$ \\
\hline 27.552 & Aromadendrene & 0.15 & - & $\mathrm{C}_{15} \mathrm{H}_{24}$ & $\mathrm{SH}$ \\
\hline
\end{tabular}


Avrupa Bilim ve Teknoloji Dergisi

\begin{tabular}{|c|c|c|c|c|c|}
\hline 27.658 & Aristolen & 1.35 & - & - & $\mathrm{OC}$ \\
\hline 27.914 & Humulen & 0.26 & - & $\mathrm{C}_{15} \mathrm{H}_{24}$ & $\mathrm{SH}$ \\
\hline 28.027 & $\beta$-Farnesene & 0.13 & - & $\mathrm{C}_{15} \mathrm{H}_{24}$ & $\mathrm{SH}$ \\
\hline 28.140 & $\alpha$. -Humulene & 5.70 & 16.14 & $\mathrm{C}_{15} \mathrm{H}_{24}$ & $\mathrm{SH}$ \\
\hline 28.269 & Alloaromadendrene & 1.34 & 0.05 & $\mathrm{C}_{15} \mathrm{H}_{24}$ & $\mathrm{SH}$ \\
\hline 28.654 & Cadina-1(6) & 0.37 & - & $\mathrm{C}_{15} \mathrm{H}_{24}$ & $\mathrm{SH}$ \\
\hline 28.740 & $\alpha$-Amorphene & 0.88 & 0.13 & $\mathrm{C}_{15} \mathrm{H}_{24}$ & $\mathrm{SH}$ \\
\hline 28.846 & $\delta$-Cadinene & 2.19 & 0.34 & $\mathrm{C}_{15} \mathrm{H}_{24}$ & $\mathrm{SH}$ \\
\hline 29.065 & Isoledene & 0.13 & - & $\mathrm{C}_{15} \mathrm{H}_{24}$ & $\mathrm{SH}$ \\
\hline 29.195 & $\beta$.-Selinene & - & 0.76 & $\mathrm{C}_{15} \mathrm{H}_{24}$ & $\mathrm{SH}$ \\
\hline 29.307 & $\alpha$. -Copaene & 0.38 & - & $\mathrm{C}_{15} \mathrm{H}_{24}$ & $\mathrm{SH}$ \\
\hline 29.431 & $\alpha .-$ Selinene & - & 0.23 & $\mathrm{C}_{15} \mathrm{H}_{24}$ & $\mathrm{SH}$ \\
\hline 29.446 & Epızonaren & 0.53 & - & $\mathrm{C}_{15} \mathrm{H}_{24}$ & $\mathrm{SH}$ \\
\hline 29.518 & $\alpha$. -Muurolene & 0.75 & 0.21 & $\mathrm{C}_{15} \mathrm{H}_{24}$ & $\mathrm{SH}$ \\
\hline 29.595 & Pentadecane & - & 0.15 & $\mathrm{C}_{18} \mathrm{H}_{38}$ & $\mathrm{AH}$ \\
\hline 29.663 & $\Delta$-Cadinene & 2.33 & 0.48 & & $\mathrm{SH}$ \\
\hline 30.288 & Cadinene & 0.32 & - & $\mathrm{C}_{15} \mathrm{H}_{24}$ & $\mathrm{SH}$ \\
\hline 30.461 & ק.-Guaiene & 0.06 & - & $\mathrm{C}_{15} \mathrm{H}_{24}$ & $\mathrm{SH}$ \\
\hline 30.840 & a.-Calacorene & 0.65 & - & $\mathrm{C}_{15} \mathrm{H}_{20}$ & AAI \\
\hline 31.476 & Cadala-1(10),3,8-Triene & 0.01 & - & $\mathrm{C}_{10} \mathrm{H}_{14}$ & $\mathrm{MH}$ \\
\hline 32.081 & Heptyl Octanoate & - & 0.11 & $\mathrm{C}_{15} \mathrm{H}_{30} \mathrm{O}_{2}$ & $\mathrm{OC}$ \\
\hline 32.110 & Cembrene & 0.04 & - & $\mathrm{C}_{20} \mathrm{H}_{32}$ & $\mathrm{OC}$ \\
\hline \multirow[t]{11}{*}{32.698} & Hexadecane & - & 0.08 & $\mathrm{C}_{16} \mathrm{H}_{34}$ & $\mathrm{AH}$ \\
\hline & & 99.93 & 99.80 & & \\
\hline & Component number & 86 & 64 & & \\
\hline & AA: Aromatik alkol & 0.02 & 3.07 & & \\
\hline & AAI: Aromatik aldehit & 1.52 & 1.70 & & \\
\hline & AH: Aromatik hidrokarbon & 2.96 & 1.50 & & \\
\hline & MH: Monoterpen hidrokarbon & 44.91 & 40.29 & & \\
\hline & OM: Oksijenli monoterpen & 0.21 & 0.23 & & \\
\hline & OC: Diğer bileşenler & 1.78 & 2.05 & & \\
\hline & SH: Seskiterpen hidrokarbon & 48.49 & 50.96 & & \\
\hline & FA: Yağ asitleri metil esteri & 0.04 & - & & \\
\hline
\end{tabular}

*"-" sembolü 0.01'den az olan bileşenler için kullanılmıştır.

*"R.T." Retention Time

\section{Discussion and Conclusion}

Helichrysum arenarium subsp. aucheri according to the results obtained from flower plants leaves and flowers, $\alpha$ - Pinene (14.79\%), (22.5\%); trans-Caryophyllene $24.33 \%, 24.57 \%$ as it is the most active in samples from its two sample areas. The same analysis is in the sample samples from $\beta$-pinene Adana (8.98\%), the main component is in the sample samples from Isparta $(6.38 \%)$ in the environment at a lower rate. $\alpha$.-Humulene and dlLimonene are the most effective applications in the samples collected from Isparta Aksu, respectively (16.14\%) and (11.98\%), while the $\alpha$-Humulene was $5.70 \%$ lower in the samples from Adana and the dl-Limonene component was not detected.

In general, the essential ingredients in Helichrysum spp. essential oil are neryl acetate (1), $\gamma$-curcumene (2), (+) limonene, neryl propionate, $\alpha$-pinene (3), ar-curcumene, italidione I (4), nerol, italicene , linalool, italidione II (5), eudesm-5-en-11-ol, italidione II isomer, 4,6-dimethyloctan-3,5dione (italidione III), 4-methylhexan-3-one, isoitalicene and 1 Reported as 8-cineole (Tisserand \& Young, 2014). It is reported that the essential oil components differ significantly due to the polymorphism in the studies conducted in the regions where it has a wide spread area. Geraniol $(36 \%)$, geranyl acetate $(15 \%)$ and nerolidol (12\%) in Greek-origin essential oils (Chinou et al., 1996), neryl acetate (36-51\%) and $\alpha$-pinene in France-Corsica essential oils (17\%) and $\gamma$-curcumene (15\%) (Bianchini et al., $2001)$, neryl acetate $(17 \%), \alpha$-pinene $(5 \%)$ and $\gamma$-curcumene $(16 \%)$ in goldgrass essential oils originating from Bosnia and
Herzegovina ( Licina and Kralj, 2016), $\alpha$-pinene, neryl acetate, $\alpha$-cedrene, nerol, ar-curcumene, $\gamma$-curcumene and geranyl acetate (Mastelić et al., 2008), in goldgrass essential oils of Croatian origin neryl acetate $(28.2 \%), \gamma$-curcumene $(18.8 \%)$, neryl propionate $(9.1 \%)$ and ar-curcumene $(8.3 \%)$ (Kladar et al., 2015), $\alpha$-pinene $(22 \%)$ in goldgrass essential oils of Serbia origin , $\gamma$-curcumene $(10 \%), \beta$-selinene $(6 \%)$, neryl acetate $(6 \%)$ and $\beta$-caryophyllene (5\%), and ar-curcumene (15-29\%) in group 1 in Adriatic goldgrass essential oils. ) and $\gamma$-curcumene (10$22 \%$ ), $\alpha$-pinene $(25-30 \%)$ and neryl acetate (4-14\%) in group 2 (Blazevic et al. , 1995).

Torabbeigi et al. (2006) In Iran H. aucheri essential oil components $\alpha$-pinene (39.6\%), 1,8-cineol (19.7\%), $\beta$ caryophyllene (7.3\%); van Vuuren (2006); In South Africa, in Asia, H. Cymosum essential oil components include $\alpha$-pinene (12.4\%), 1,8-cineole (20.4\%), $\beta$-Caryophyllene (10.8\%); H. gymnocephalum 151,8 -cineole (17\%), borneol (16\%), ItalyCaryophyllene (13\%); Charles, D.J., Simon, J.E. (1991); In North Africa, H. italicum subsp. Nerylacetate (51.4\%), $\alpha$-pinene $(17 \%)$, North African neryl acetate (17\%), $\gamma$-curcumene $(16 \%)$ in Italicum essential oil components; Tsoukatou et al., (1999) Spain H. stoechas ssp. Stoechas blown up $\alpha$-pine (28.3\%), epi- $\alpha$ bisabolol $(21.9 \%)$, Spain $\beta$-karyophyllene $(5.5 \%)$ as the main target.

Solid-phase micro-extraction (SPME) method, which is used to obtain volatile components, was used in this study. The SPME method has become a highly preferred method that provides sensitivity with ease of use with fast and low cost sampling without solvent (Umaz et al., 2019). In this study, the volatile 
components of gold grass from two different locations were determined and compared with the SPME / GC-MS method. In our research, H. arenarium ssp. The $\alpha$-pinene component, which we identified as one of the most active components in our aucheri sample, was also found at high rates in studies conducted in literature, while other components determined in the literature were found at lower rates in our study. According to the studies carried out, some differences were determined in terms of the essential oil basic components and ratios of the tested plant species compared to other studies. When we look at the literature, the $\alpha$-pinene component, which is one of the most active components we found in the experimental study, has antimicrobial (Moslemi et al.2012), anti-inflammatory, antibacterial, antioxidant, anticancer and antinociceptive activities (Aydin et al., 2013) and the anti-inflammatory component of the trans-Caryophyllene component (Fernandes at all., 2006), we can say that this plant can be used effectively in the pharmaceutical industry because it shows activity.

In addition, the variety and amount of bioactive substances present in medicinal and aromatic plants may also differ according to the part of the plant used, post-harvest processes, and the methods of obtaining and analyzing the essential oil used. Considering that essential oil components differ according to environmental factors, more studies should be carried out on different types of gold from different locations in our country, which are rich in gold grass species, in order to reveal the chemical profile of Helichrysum spp. species.

\section{References}

Aftab, T. 2019. A review of medicinal and aromatic plants and their secondary metabolites status under abiotic stress. Journal of Medicinal Plants, 7(3), 99-106.

Anderberg, A. A. 1991. "Taxonomy and phylogeny of the tribe Gnaphalieae (Asteraceae)", Opera Botanica 104, 1-195.

Angioni, A., Barra, A., Arlorio, M., Coisson, J. D., Russo, M. T., Pirisi, F. M., Satta, M., Cabras, P. 2003. "Chemical composition, plant genetic differences, and antifungal activity of the essential oil of Helichrysum italicum G. Don ssp. microphyllum (Willd) Nym", Journal of Agricultural and Food Chemistry, 51(4), 1030-1034.

Appendino, G., Ottino, M., Marquez, N., Bianchi, F., Giana, A., Ballero, M., Sterner, O., Fiebich, L., Munoz, E. 2007. "Arzanol, an anti-inflammatory and anti-HIV-1 phloroglucinol a-pyrone from Helichrysum italicum ssp. microphyllum", Journal of Natural Products, 70, 608-612.

Araujo, H.C., Lacerda, M.E.G., Lopes, D., Bizzo, H.R., Kaplan, M.A.C., 2007. Studies On The Aroma Of Mate (Ilex paraquariensis St.Hil.) Using Headspace SolidPhase Microextraction. Phtochemical Analysis, 18: 469- 474.

Aydin E, Türkez H, Geyikoğlu F. 2013. Antioxidative, anticancer and genotoxic properties of $\alpha$-pinene on $\mathrm{N} 2 \mathrm{a}$ neuroblastoma cells. Biologia, 68(5): 1004-1009.

Battaglia S. 2003. The Complete Guide to Aromatherapy, $2^{\text {nd }}$ edition. 624 p, Brisbane: The International Centre of Holistic Aromatherapy.

Bayaz, M. 2014. Esansiyel yağlar: antimikrobiyal, antioksidan ve antimutajenik aktiviteleri. Academic Food Journal/Akademik G1da, 12(3).

Bayram, E., Kırıcı, S., Tansı, S., Yılmaz, G., Arabacı, O., Kızıl, S., Telci, D., 2010. "Tibbi Ve Aromatik Bitkiler Üretiminin Arttırılması Olanakları”. Türkiye Ziraat Mühendisliği VII.
Teknik Kongresi Bildiriler Kitabı-I, 437-456, 11- 15 Ocak, Ankara.

Baytop T. Türkçe Bitki Adları Sözlüğü. Türk Dil Kurumu Yayınları. Ankara; 1997.

Bianchini, A., Tomi, P., Costa, J., Bernardini, A. F. 2001. "Composition of Helichrysum italicum (Roth) G. Don fil. subsp. italicum essential oils from Corsica (France)", Flavour and Fragrance Journal, 16, 30-34.

Blažević, N., Petričić, J., Stanić, G., Males Z. 1995. "Variation in yields and composition of immortelle (Helichrysum italicum Roth Guss.) essential oil from different locations and vegetation periods along Adriatic coast”, Acta Pharm.,45, $517-522$.

Catty, S. 2001. Hydrosols: the next Aromatherapy. Vermont: Healing Arts Press. 96-97.

Charles, D.J., Simon, J.E. (1991). Volatile compounds of the curry plant, Hort Science, 26: 69- 70.

Chinou, I.B., Roussis,V., Perdetzoglou, D., Loukis, A. 1996. "Chemical and biological studies on two Helichrysum species of Greek origin”, Planta Med. ; 62(4), 377.

Christaki, E., Bonos, E., Giannenas, I., Florou-Paneri, P. 2012. Aromatic plants as a source of bioactive compounds. Agriculture, 2(3), 228-243.

Davis, P. H., Mill, R. R. \& Tan, K. (eds.) 1988: Flora of Turkey and the East Aegean Islands 10. - Edinburgh.

Davis, P. H., R. R. Mill, and K. Tan. 1988. Flora of Turkey and the East Aegean Islands, 96-103. Edinburgh, UK: Edinburgh Univ. Pres.

Dönmez, İ.E., Salman, H., 2017. Yaban mersini (Myrtus communis L.) yaprak ve meyvelerinin uçucu bileşenleri. Turkish Journal of Forestry, 18(4): 328-332.

Eroğlu, H. E. 2018. The Names in Turkish and Other Languages of Turkey Helichrysum Taxa. Avrasya Terim Dergisi 6 (1):26-34.

Fernandes Elizabeth S. a, Giselle F. Passos a, Rodrigo Medeiros a, Fernanda M. da Cunha a, Juliano Ferreira b, Maria M. Campos c, Luiz F. Pianowski d, João B. Calixto.2006. Antiinflammatory effects of compounds alpha-humulene and (-)trans-caryophyllene isolated from the essential oil of Cordia verbenacea European Journal of Pharmacology 569 (2007) 228-236.

Gattefossé, R. M. 1993. Gattefossé Aromatherapy. UK: The C.W. Daniel Company. p25.

Guner, A., N. Ozhatay, T. Ekim, and K. H. C.Baser. 2000. Flora of Turkey and the East Aegean Islands.Edinburgh: Edinburgh University Press," 11, 2000.

Haas, M. 2004. Quick Reference Guide for 114 Important Essential Oils. San Rafael, CA: Linda Scent and Image books.

Harris, B. Harris, R. 2002. Aromatherapy for Pain Relief. Retrieved on October 28.

Igwillo U.C, Ola-Adedoyin A. T, Abdullahi M. M And Chukwuemeka A. E. 2019. A review of opportunities and challenges in conservation and use of medicinal and aromatic plants in Nigeria. International Journal of Advanced Research, 7 (4). 770-778 (ISSN 2320-5407).

Kayaalp S.O. 2001. Rasyonel Tedavi Yönünden Tibbi Farmakoloji, 1.cilt, 9. baskı, Hacettepe-TaG̣ Kitapçılık, Ankara, sayfa: 19, 21-22, 25-26, 175.

Kladar, N.V., Anačkov, G.T., Rat, M. 2015. "Biochemical Characterization of Helichrysum italicum (Roth) G.Don subsp. italicum (Asteraceae) from Montenegro: Phytochemical Screening, Chemotaxonomy, and 
Antioxidant Properties”, Chemistry \& Biodiversity, 12(3), 419-431.

Lawless, J. 2002. The Encyclopedia of Essential Oils. London: Thorsons. p107.

Licina, A., Kralj, V.R. 2016. "Mediterranean Gold-Helichrysum italicum", The International Journal of Professional Holistic Aromatherapy, 4(4), 5-12.

Marković, S. 2005. Fitoaromaterapija. Zagreb: Centar Cedrus. p245.

Mastelić, J., Politeo, O., Jerković, I. 2008. "Contribution to the analysis of the essential oil of Helichrysum italicum (Roth) G. Don. determination of ester bonded acids and phenols", Molecules, 13(4), 795-803.

Millou, Y., Fontes, K., Tourel, C. 2010. Cosmetic composition comprising an essential oil, extracted from Helichrysum italicum. United States Patent, No: US7666454B2 dated 23.02.2010.

Mojay, G. 1996. Aromatherapy for Healing the Spirit. London: Gaia Books Limited, p:70-71.

Moslemi H.R,Hoseinzadeh H, Badouei M.R et al. Antimicrobial Activity of Artemisia absinthium Against Surgical Wounds Infected by Staphylococcus aureus in a Rat Model. Indian J Microbiol 2012;52:601-604.

Nostro, A., Bisignano, G., Cannatelli, M. A., Crisafi, G., Germano, M. P., Alonzo, V. 2001. "Effects of Helichrysum italicum extract on growth and enzymatic activity of Staphylococcus aureus", International journal of antimicrobial agents, 17(6), 517-520.

Peyron, L., Roubaud, M. 1971. Parfums, Cosmetiques et Savons de France, p: 129.

Poštić, S. 2013. V čarobnem svetu vonjev. Ljubljana: Buča. p93.

Price, S., Price, L. 2012. Aromatherapy for Health Professionals, $4^{\text {th }}$ ed. London: Churchill Livingstone 315.

Roussis, V., Tsoukatou, M., Chinou, I.B., Ortiz, A. 1998. "Composition and antibacterial activity of the essential oils of Helichrysum rupestre and H. ambiguum growing in the Balearic Islands1 (Part III)", Planta medica, 64(07), 675-676.

Sala, A., Recio, M., Giner, R.M., Máñez. S., Tournier, H., Schinella, G., Ríos, J.L. 2002. "Anti-inflammatory and antioxidant properties of Helichrysum italicum", Pharm Pharmacol. ,54 (3), 365-371.

Schnaubelt, K. 2011. The Healing Intelligence of Essential Oils. Toronto: Healing Art Press. p152-155.

Sümbül, H., R. S. Göktürk, and O, and D. Düşen. 2003. A new endemic species of Helichrysum Gaertn. (AsteraceaeInuleae) from south Anatolia. Botanical Journal of the Linnean Society 141:251-54. doi:10.1046/j.10958339.2003.00109.x.

Şen N., Kalaycı G. 2016. Altın Otu Bitkisinden (Helichrysum arenarium) Tanen ve Kumarinin Kimyasal Kompozisyonu 42 (2): 226-231.

Tabata M, Honda G, Sezik E et al. 1993. A report on traditional medicine and medicinal plants in Turkey 1990, 1991. Faculty of Pharmaceutical Sciences, Kyoto University. Kyoto, s. 54 - 107

Tisserand R, Young R. 2014. Essential oil safety. $2^{\text {nd }}$ ed. Edinburgh: Churchill Livingstone, Elsevier.

Torabbeigi M., Azar P.A. \& Meibodi Z. A. 2006. Chemical Composition of Essential Oils of Aerial Partsof Helichrysum aucheri from Iran. Analytical Chemistry Letters. 1:5-6, 393396, DOI: 10.1080/22297928.2011.10648243

Tsoukatou, M., Roussis, V., Chinou, L., Petrakis, P.V., Ortiz, A. (1999). Chemical Composition of the Essential Oils and
Headspace Samples of Two Helichrysum Species Occurring in Spain, Journal of Essential Oil Research, 11: 511-516.

Van Vuuren, S. F., du Toit, L. C., Parry, A., Pillay, V., Choonara, Y. E. (2010). Encapsulation of essential oils within a polymeric liposomal formulation for enhancement of antimicrobial efficacy. Natural product communications, 5(9), 1401-1408.

Van Vuuren, S.F., Viljoen, A.M., van Zyl, R.L., van Heerden, F.R., Baser, K.H.C. (2006). The antimicrobial, antimalarial and toxicity profiles of helihumulone, leaf essential oil and extracts of Helichrysum cymosum (L.) D. Don subsp. cymosum, South African Journal of Botany, 72: 287-290.

Zeybek, U. and N. Zeybek. 2002. Farmasötik Botanik. 3. Bask1, E.Ü. Ecz. Fak. Yayın. No.3, Ege Üniversitesi Basımevi, Bornova-İzmir, pp. 378-382. 